\title{
ESTUDIO DE LA HACIENDA MUNICIPAL A TRAVÉS DE LOS LIBROS DE PROPIOS DE LA CIUDAD DE VILLENA (1708-1766)
}

\author{
Por José A. MORENO NIEVES
}

\section{INTRODUCCIÓN}

El tema que aquí tratamos, la hacienda municipal, ha sido quizá uno de los que menos se ha escrito por la moderna historiografía española sobre el Antiguo Régimen. A ello ha contribuido, sin duda, la falta de fuentes precisas sobre el tema y la dificultad del tratamiento de las mismas, pero el hallazgo de los denominados «libros de propios» en el Archivo Municipal de Villena nos indujo a introducirnos en su estudio ${ }^{(1)}$. Quedan en ellos recogidas todas las cuentas efectuadas anualmente por el mayordomo de propios desde el año 1708 a 1766, no encontrándose únicamente las de 1748, 1755, 1761 y 1762, y que aparecen en cinco tomos.

Una primera cuestión que se nos planteó a la hora de estudiar el desconocido tema de las haciendas municipales fue su definición. ¿Qué debe entenderse por hacienda municipal en el Antiguo Régimen? Es evidente que éstas no aparecen tan claramente delimitadas como en la actualidad por diversos motivos. El primero de ellos es la difícil distinción entre hacienda municipal y hacienda real, motivada por el alto grado de intervencionismo que los mecanismos estatales de fiscalidad habían introducido en el municipio, lo que se hace aún más patente en el siglo XVIII. Así el municipio se había convertido en un agente real recaudatorio tras el establecimiento en la primera mitad del siglo XVI del sistema de los encabezamientos. Por otra parte un segundo grupo de dificultades existen a la hora de definir la hacienda municipal en el Antiguo Régimen: se trata de la peculiar forma de administración de los principales efectos de ésta, como eran los propios y los arbitrios, a través de un funcionario municipal, el mayordomo, en el que quedaba personificada dicha hacienda, no existiendo un presupuesto previo, ni la distribución del gasto racionalizado, sino al socaire del momento más oportuno para distribuir los ingresos. 
Tradicionalmente dos han sido los componentes que se consideraban fundamentales para la delimitación de la hacienda municipal en el Antiguo Régimen: los propios y los arbitrios. En el caso de Villena podemos concluir que ésta se encontraba básicamente compuesta por los propios, ya que durante el siglo XVIII no existe ningún arbitrio municipal, aunque se atestiguan en otras épocas. Así lo indican expresamente los componentes del cabildo municipal en $1735^{(2)}$ y en 1755 en la respuesta 24 al cuestionario para el establecimiento de la Única Contribución ${ }^{(3)}$.

Sin embargo pueden considerarse otras formas de subvenir los gastos municipales como eran la concesión extraordinaria del recobro de parte de las rentas o impuestos reales o el reparto directo entre el vecindario de algún gasto como el salario de los médicos y el maestro o el gasto efectuado en la extinción de la plaga de langosta en 1757. Pero aquí nos dedicaremos exclusivamente al principal componente de la hacienda de Villena: los propios.

\section{LOS BIENES DE PROPIOS DE VILLENA EN LA PRIMERA MITAD DEL SIGLO XVIII}

Los bienes de propios del municipio de Villena durante el siglo XVIII tienen un carácter diverso que debe ser tenido en cuenta a la hora de conocer la evolución general de los ingresos por los propios. Hemos agrupado estos bienes en cinco grupos según el carácter económico y el origen de los mismos.

En el apartado de rentas inmobiliarias agrícolas aparecen siete dehesas (Alorines, Campo, Carboneras, Salinas, Puerto, Moratillas y Hoya de Alpañés) que se fueron incorporando a los propios a lo largo de los siglos XVI y XVII; tres bancales de regadío (dos en la partida del Hilo del Rey y uno en la partida de la Balsa de la Alcudia) que totalizan 18 tahúllas, lo que significa tan sólo el 0,24 por ciento del regadío de Villena.

Un carácter distinto tienen otras tres rentas municipales que se derivan de la recogida de tres productos de crecimiento espontáneo en el término como eran la hierba salada, el junquillo y la fruta de los pinares. La recogida de la hierba salada (barrilla y salicornio) comenzó a reportar beneficios al concejo en el segundo cuarto del siglo XVI. El junquillo parece tratarse de una renta ocasional que durante el siglo XVIII sólo se arrienda desde 1730 a 1744, no atestiguándose en épocas anteriores. La recogida de la piña (conocida como «fruta del pinar doncel»), se basaba en la propiedad municipal de todos los pinares del término, aun aquellos que estuviesen dentro de propiedades particulares, desde época medieval.

Un segundo apartado lo componen lo que hemos denominado rentas inmobiliarias agrícolas que proceden del arrendamiento de propiedades cuyo carácter económico está ligado a las actividades secundarias y terciarias. Un hecho a destacar en este grupo de rentas municipales es su reciente incorporación a los bienes de propios, puesto que ninguna de ellas aparece con anterioridad a 1708, si no que se van incorporando paulatinamente a las rentas municipales. 
Se trata del horno, arrendado por primera vez en 1711, los puestos de la feria franca de septiembre, que se inicia en 1708, tras la aprobación real el 14 de septiembre de 1707 , pero que no comienza a reportar beneficios al municipio hasta 1711 , las llamadas «tiendas de especiería y saladura», que comenzó a arrendarse en 1717, el molino de propiedad municipal, situado junto al convento agustino de Nuestra Señora de las Virtudes, y que recibía el agua necesaria para su funcionamiento de la llamada Fuente del Chopo, que comenzó a arrendarse en 1724, las balsas del cáñamo, que, tras un proceso de apropiación y desalojo de las de propiedad privada por motivos de salud pública, comenzaron a reportar beneficios al concejo en 1742. Por último englobamos en este apartado algunas casas que, tras su arrendamiento, producían ingresos a los propios, sin embargo, en ellas destaca el hecho de que su arrendamiento no es continuado, ya que en algunos períodos son ocupadas por funcionarios municipales (pregonero o porteros) sin pagar un alquiler al municipio.

El siguiente apartado lo componen las rentas percibidas por el concejo mediante el arrendamiento o administración de algunos servicios públicos, por los que o bien el vecindario tenía que contribuir, caso de administrarse directamente por el concejo, o por los que un arrendatario, servidor de este oficio tenía que satisfacer un cierto precio.

Uno de ellos es el llamado «acequiaje», que se trata de una de las rentas más antiguas del concejo, puesto que ya aparece mencionado en documentos de 1491 . Se trataba en realidad de un impuesto sobre las propiedades de la huerta, ya que debía satisfacer cada poseedor de tierras de regadío un celemín de trigo o su equivalente en dinero, por cada bancal regado. Hasta 1726 el sistema más común es el del arrendamiento de este impuesto, siguiendo el mismo sistema que para el cobro de los impuestos reales efectuaba la Corona. Pero desde 1727, como consecuencia de la promulgación de las «Ordenanzas de Aguas» por Felipe V y la creación en ellas del cargo de Alcalde o Juez de Aguas entre los regidores, pasa el municipio a cobrarlo directamente a los propietarios de la huerta de Villena.

Otros servicios públicos incorporados a los propios eran la escribanía pública, que fue otorgada por los Reyes Católicos en el año 1500, pero que fue origen de numerosos pleitos entre los escribanos, apoyados por la Corona, y el municipio, ya que los primeros pretendían ejercerla sin pagar una renta al municipio lo que consiguieron «de facto» desde 1742, fecha desde la que dejan de pagar su importe, aunque el municipio sigue considerándola como parte de sus ingresos. También reportaba ingresos al municipio el disfrute del oficio de la almotazanía y correduría, que era sacado a pública subasta anualmente, siendo uno de los ingresos más antiguos que se remonta a los inicios del siglo XVI, aunque sólo pasa a formar parte de las cuentas de propios en 1719, ya que con anterioridad el importe de su arrendamiento se dedicaba directamente al pago del salario de los porteros. Un último apartado de los servicios públicos era el denominado de «livianos», que consistía en el importe de la venta de parte de las reses que los arrendadores de la dehesa carnidera, dedicada al abastecimiento de la ciudad, dejaban al municipio en concepto de pago por su disfrute. 
Otro apartado está compuesto por los censos percibidos por el concejo y agregados a los propios. Estos censos pueden clasificarse según la entidad que los satisfaga (una villa o un particular) o el origen del mismo (donación, venta de agua del término o por préstamo de caudales públicos). El disfrute del agua del manantial de la Fuente del Chopo por las villas de Elda y Elche dio lugar a la aparición de dos censos pagados por éstas, el primero por contrato estipulado en 1656 y el segundo en fecha sin determinar durante el último cuarto del siglo XVII. Pero el primer contrato por este agua se remonta al año 1505. fecha en la aue el 
to que las partidas de propios no cobradas las incorporaba al año siguiente dentro del cargo de fincas y en el año en curso dentro de la llamada data de fincas, para que al realizar el balance de lo que le sobraba o se le adeudaba al mayordomo no existiesen problemas.

En la segunda parte de las cuentas de propios aparecían los gastos bajo el epígrafe de data, tras la que se incluían los correspondientes recibos de los gastos.

Por ello para conocer los ingresos efectivos de un año determinado no sólo hay que tener en cuenta el llamado cargo, ya que pueden existir partidas que no se hayan podido cobrar por el mayordomo. Para ello hay que comprobar si en la data de fincas existe alguna partida correspondiente a ese año, y para más seguridad comprobar si en el cargo de fincas del año siguiente existe la obligación del mayordomo de cobrar alguna deuda del año precedente.

En primer lugar vamos a realizar un análisis de la evolución de los propios agrupados en las cinco clases a que hicimos referencia en el punto anterior para finalizar este apartado con unas notas sobre la evolución general de los mismos. Todo ello agrupado por quinquenios debido a las limitaciones del presente trabajo ${ }^{(5)}$.

\subsection{Evolución particular de los grupos de bienes de propios}

a) Las rentas por las propiedades inmobiliarias agrícolas.

No creemos que sea preciso hacer constar la importancia que dentro de la economía del Antiguo Régimen tenían las actividades agropecuarias, pero para entender en su justo punto el desarrollo de las rentas municipales provenientes de estas actividades es necesario hacer notar algunas características de las mismas en Villena.

Para ello debe tenerse en cuenta la importancia de cada uno de los bienes de propios dentro del conjunto de las rentas por las propiedades inmobiliarias agrícolas, para poder explicarnos posteriormente a qué se deben las variaciones de estos ingresos.

El predominio de los ingresos por el arrendamiento de las dehesas es notorio; sobre el conjunto de nuestra serie (1708-1766) suponen el 76,68 por ciento. Por ello deberemos atender al movimiento de éstos para explicarnos en gran medida las fluctuaciones de las rentas inmobiliarias agrícolas. El segundo lugar lo ocupan los ingresos por el arrendamiento de la hierba salada, que supone el 10,54 por ciento del total de este grupo. A continuación se sitúa el arrendamiento de la fruta del pinar doncel con el 6,86 por ciento, seguido del arrendamiento de los bancales de regadío con el 5,77 por ciento, lo que nos da a conocer la escasa importancia de estas propiedades en el conjunto de los propios. Una renta marginal como dijimos era el arrendamiento de la recogida del junquillo, que sólo representa el 0,12 por ciento, debido a que sólo se arrienda durante un corto período de nuestra serie.

La primera característica de estos ingresos de propios es su escaso crecimien- 
to con una tasa de crecimiento anual del 0,62 por ciento, que es la más baja de todos los grupos de ingresos de propios. Otra característica de estos ingresos es su escasa movilidad, es decir las pocas alteraciones de los datos respecto de la media, lo que se cuantifica en el coeficiente de variación interanual ${ }^{(6)}$, que es del 18,81 por ciento, que es el menor de todos los ingresos de propios.

En el Cuadro I podemos observar que, a pesar de las escasas variaciones producidas, existe un primer período estable (1708-22) hasta que se produce el primer crecimiento importante en 1723-27, pero que es seguido por un descenso casi similar en el siguiente quinquenio. Una de las características principales del cuadro que recoge el desarrollo de estos ingresos es que un crecimiento quinquenal se ve compensado por un descenso en el quinquenio siguiente, lo que da a los datos un movimiento pendular y esa tasa de crecimiento tan escasa.

\section{CUADRO I}

\section{EVOLUCIÓN DE LAS RENTAS AGRÍCOLAS}

\begin{tabular}{|c|c|r|r|}
\hline PERIODO & REALES & INDICE (7) & TASA DE CRECIMIENTO \\
\hline $1708-12$ & 24.710 & 82,49 & - \\
$1713-17$ & 25.543 & 85,28 & 0,33 \\
$1718-22$ & 25.385 & 84,75 & $-0,66$ \\
$1723-27$ & 30.777 & 102,75 & 1,94 \\
$1728-32$ & 27.722 & 92,55 & $-1,03$ \\
$1733-37$ & 33.068 & 110,40 & 1,77 \\
$1738-42$ & 31.854 & 106,35 & $-0,37$ \\
$1743-47$ & 28.374 & 94,73 & $-1,15$ \\
$1749-53$ & 34.683 & 115,79 & 2,02 \\
$1754-59$ & 37.277 & 124,45 & 0,72 \\
$1760-66$ & 30.076 & 100,41 & $-2,12$ \\
\hline TOTAL........ & 329.469 & & 0,62 \\
\hline
\end{tabular}

Fuente: A. M. V. «Libros de Propios» (elaboración propia).

Todo ello se enmarca en la dinámica que las rentas agrícolas suelen presentar en el Antiguo Régimen, debido a la inseguridad de la producción. Aún más en el caso de las rentas percibidas por el municipio de Villena ya que las propiedades de regadío tienen una escasa importancia, predominando las rentas por las dehesas, ligadas al desarrollo de la ganadería, que ya había perdido la mayor parte de su empuje en el siglo XVIII.

Por lo tanto el incremento que se produce en el arrendamiento de la hierba salada no se ve muy reflejado en el conjunto de las rentas agrícolas al no representar un porcentaje importante en las mismas. Esta renta presentaría un movi- 
miento más acorde con el que aparece en la mayoría de las rentas por las propiedades municipales no agrícolas y ligadas a los sectores secundario y terciario de la economía, puesto que este producto se dedicaba en su mayoría a la exportación por los puertos de Alicante y Cartagena.

Del mismo modo que las dehesas, tampoco el arrendamiento de la fruta del pinar doncel podía tener un gran desarrollo por las pérdidas que en este arbolado se habían producido sobre todo desde el final de la Guerra de Sucesión para reponer la mayoría de los edificios públicos y algunos privados.

En cuanto a los bancales ya hemos señalado su escasa importancia en el conjunto de las rentas agrícolas, aunque es preciso señalar el incremento que se produce a finales del período tratato. El escaso desarrollo que hasta esa época había manifestado se debe a la acaparación que de los dos bancales situados en el Hilo del Rey había hecho Pedro López Martínez, mayordomo de propios (1745-49) entre los años 1730-1766.

b) Las rentas por las propiedades inmobiliarias no agrícolas.

Si los ingresos por las rentas agrícolas hemos visto que apenas si crecen durante las primera mitad del siglo XVIII, los ingresos que el municipio percibe por las propiedades inmobiliarias no agrícolas experimentan un fuerte crecimiento en el mismo período. La tasa de crecimiento de estos ingresos asciende al 4,87 por ciento, frente al 0,62 por ciento de las anteriores, y superior al del conjunto de los ingresos de propios que es del 3,42 por ciento.

\section{CUADRO II \\ EVOLUCIÓN DE LOS INGRESOS POR LAS PROPIEDADES INMOBILIARIAS NO AGRÍCOLAS}

\begin{tabular}{|c|r|r|c|}
\hline PERIODO & REALES & INDICE & TASA DE CRECIMIENTO \\
\hline $1708-12$ & 4.652 & 9,07 & - \\
$1713-17$ & 11.902 & 23,21 & 0,23 \\
$1718-22$ & 25.422 & 49,58 & 7,88 \\
$1723-27$ & 32.713 & 63,80 & 2,55 \\
$1728-32$ & 41.093 & 80,15 & 2,30 \\
$1733-37$ & 44.209 & 86,22 & 0,73 \\
$1738-42$ & 52.433 & 102,26 & 1,72 \\
$1743-47$ & 63.401 & 123,66 & 1,91 \\
$1749-53$ & 69.632 & 135,81 & 0,94 \\
$1754-59$ & 86.004 & 167,74 & 2,13 \\
$1760-66$ & 101.742 & 198,44 & 1,69 \\
\hline TOTAL $\ldots . . . . .$. & 533.203 & & 4,87 \\
\hline
\end{tabular}

Fuente: A. M. V. «Libros de Propios» (elaboración propia). 
Este crecimiento espectacular está motivado por diversas causas. Una de ellas es el evidente aumento que experimentan los arrendamientos de estos efectos inmobiliarios no agrícolas a lo largo del período que estudiamos. Estas propiedades parece que eran cada vez más apetecidas por los individuos que tenían suficientes caudales para arrendar estos efectos; por lo tanto la presión sobre ellos parece que fue cada vez mayor, lo que hizo crecer el precio de los arrendamientos.

Una segunda causa que hace crecer los ingresos por las propiedades inmobiliarias no agrícolas es la incorporación paulatina a lo largo del siglo de nuevas propiedades. De este modo el crecimiento espectacular que se produce en el período 1718-22 se debe a la incorporación de la tienda de especiería y saladura.

Sin embargo existe una gran variación interanual en estos ingresos, constatado en su tasa de variación $(56,97 \%)$, que es superior a la del conjunto de los ingresos de propios $(40,15 \%)$ y el más alto de los grupos de ingresos, excepto los censos. Esto se debe a la inseguridad que presentan algunos de estos ingresos, que se encuentran ligados a la coyuntura internacional como las balsas del cáñamo, la tienda, que necesita para el abastecimiento de especias y salazones la llegada puntual de suministros a los puertos cercanos o los puestos de la feria de septiembre.

$\mathrm{Al}$ igual que con las rentas agrícolas, es interesante conocer la importancia de cada una de las rentas por las propiedades inmobiliarias no agrícolas. La más importante es la tienda de especiería y saladura $(43,99 \%)$ lo que nos indica hasta qué punto estos productos tenían una alta estimación en el mercado, por lo que el arrendamiento de la misma era muy apetecido y como consecuencia de ello se convirtió en una de las rentas de propios más importantes.

El segundo lugar lo ocupaba el arrendamiento de las balsas del cáñamo, que como se recordará no comenzaron a reportar beneficios al municipio hasta 1742 , lo que nos indica la importancia que éstas adquirieron desde su inicio, puesto que en los pocos años en que se arriendan (1742-1766) de nuestro período llegan a suponer el 30,73 por ciento del total de ingresos por este grupo de propiedades en toda nuestra serie. Como hecho revelador indicaremos que en el quinquenio 1754-59 suponen el 53,60 por ciento de las rentas por las propiedades inmobiliarias no agrícolas.

El resto de rentas de este grupo tienen una importancia mucho menor, puesto que las dos anteriores ya abarcan tres cuartos de estos ingresos. Así el arrendamiento del molino sólo supone el 10,49 por ciento, cuando la importancia económica de este efecto de propios nos hubiera hecho concederle una mayor participación en las rentas no agrícolas. Aparte de la importancia que adquieren los otros bienes hay que señalar como causa de este escaso desarrollo de los ingresos por el molino las dificultades que su funcionamiento presentó a lo largo de nuestro periodo, sobre todo, por la falta de agua.

El siguiente lugar lo ocupa el arrendamiento de los puestos de la feria, que tuvo mayor importancia en un principio, pero que, tras la aparición del comercio estable en Villena, decreció considerablemente para suponer sólo el 7,49 por ciento sobre el conjunto de la serie. Un porcentaje parecido $(6,81 \%)$ presentan los 
ingresos por el arrendamiento del horno, cuya escasa importancia puede relacionarse con el control que el municipio y la Corona ejercían sobre el precio de un bien tan necesario como el pan. Una renta residual es el apelativo que podemos dar al arrendamiento de las casas $(0,46 \%)$, debido a que permanecen períodos dilatados de tiempo sin arrendar al ofrecerse gratuitamente a algunos oficiales municipales.

c) Las rentas por los servicios públicos.

El conjunto de los servicios públicos no ofrecen a la hacienda municipal de Villena un gran número de ingresos. En el período de nuestra serie (1708-1766) los servicios públicos sólo reportan el 15,03 por ciento del total de los ingresos de propios.

A pesar de todo, el total de ingresos por este sector de los propios sufre un crecimiento moderado entre 1708 y 1766 , pero no se produce de manera progresiva, como sucedió con las rentas inmobiliarias no agrícolas, sino que está provocado por bruscos crecimientos al alza, debidos a peculiaridades en alguno de sus componentes.

\section{CUADRO III}

EVOLUCIÓN DE LOS INGRESOS POR SERVICIOS PÚBLICOS

\begin{tabular}{|c|r|r|r|}
\hline PERIODO & \multicolumn{1}{|c|}{ REALES } & \multicolumn{1}{|c|}{ INDICE } & TASA DE CRECIMIENTO \\
\hline $1708-12$ & 5.255 & 36,07 & \\
$1713-17$ & 9.948 & 68,29 & 6,58 \\
$1718-22$ & 12.700 & 87,19 & 2,47 \\
$1723-27$ & 18.426 & 126,50 & 3,79 \\
$1728-32$ & 15.419 & 105,85 & $-1,76$ \\
$1733-37$ & 17.357 & 119,16 & 1,19 \\
$1738-42$ & 17.305 & 118,80 & $-0,02$ \\
$1743-47$ & 10.339 & 70,98 & $-5,02$ \\
$1749-53$ & 18.956 & 130,14 & 6,24 \\
$1754-59$ & 17.494 & 120,10 & $-0,79$ \\
$1760-66$ & 17.025 & 116,88 & $-0,27$ \\
\hline TOTAL ......... & 160.224 & & 1,06 \\
\hline
\end{tabular}

Fuente: A. M. V. «Libros de Propios» (elaboración propia).

Este crecimiento moderado (1,06\% anual) está provocado por la estabilización que en estos ingresos se produce desde finales de la década de 1720 . Sin embargo se producen bruscos movimientos tanto al alza como a la baja, pero que no afectan al conjunto general pues se pasa de un índice 126,50 en el quinquenio 1723-27 a 116.88 en el último (1760-1766). Ello se debe a que tanto un ascenso 
acusado como un descenso se ve compensado, casi con la misma cuantía, en el período siguiente.

El progresivo interés que despiertan para el municipio las rentas no relacionadas directamente con las actividades agropecuarias se ve reflejado de nuevo en el nivel de participación de cada uno de los componentes de los servicios públicos.

Ello se demuestra en la gran importancia que adquieren los ingresos por la almotazanía, que suponen el 40,31 por ciento. Este servicio público debe relacionarse evidentemente con las actividades del sector terciario, pero también con el desarrollo agrícola al dedicarse no sólo al control de los pesos y medidas sino también a la medición de tierras.

El segundo lugar lo ocupaba la renta de los livianos con el 27,88 por ciento y también queda dentro de las actividades terciarias pues, si bien, también se relaciona con la ganadería, no cabe duda de que el precio de la carne es el que más posibilita el incremento de esta renta.

El resto de componentes de este grupo de bienes de propios tienen una importancia mucho menor, puesto que los dos anteriores, como en los otros grupos hasta ahora analizados, ya suponen dos tercios del total. La renta del acequiaje, a pesar de lo importante que debiera parecer, por estar impuesta sobre el regadío, no reporta grandes beneficios al municipio. El problema no se deriva de las dificultades o grado de impago sino por la apropiación que de parte de su importe hace la oligarquía local integrante del cabildo de regidores; ello se produce porque desde 1727 el Ayuntamiento pasa a administrar directamente esta renta por medio del llamado juez o alcalde de Aguas, que, sin embargo, se apropia gran parte de ella ya que se señala un salario de 80 ducados, es decir, más del 50 por ciento de lo que anualmente rendía.

El último lugar entre los servicios públicos lo ocupa la renta de la escribanía, debido, sobre todo, al alto grado de impago de los propietarios de la escribanía, ya que ésta no salía a arrendamiento anual, debido a un conflicto sobre su propiedad que arranca de mediados del siglo XVI. Ello derivó finalmente en el impago total desde 1742, aunque el municipio siguió considerándola como propia, como demuestra el que siga apareciendo en las cuentas de propios, aunque aparece siempre en el cargo de Propios como deuda a cobrar.

d) Las rentas por los censos.

La principal característica de estos ingresos es su gran variación a lo largo del período que tratamos. Este hecho está motivado no por el crecimiento o descenso de cada uno de los componentes, sino, más bien, por la aparición o no de cada uno. Sólo los censos de los particulares son pagados todos los años, mientras que el censo que pagaba Caudete sólo aparece entre 1722-36 y los que pagaban las villas de Elda, Elche y Sax por el agua de la Fuente del Chopo son pagados con gran discontinuidad.

Dentro de esa clasificación que hicimos sobre los censos es importante conocer el peso de cada uno sobre el total. El primer tipo de censos es el pagado por los vecinos de Villena tras la concesión de préstamos por parte de los caudales 
municipales. Ocupan el 40,30 por ciento del total del volumen de los censos que perciben los propios de Villena. El que ocupen el primer lugar está motivado por dos causas fundamentales: la primera es el alto índice de impago que se produce en el otro tipo de censos más duradero en el tiempo (los cargados sobre el agua de la Fuente del Chopo), pues el otro apenas sí ocupa quince años. Pero el segundo motivo es el alto índice de pago de estos censos (el 71,43 por ciento de lo que se debía haber cobrado en el período 1708-1766), que es mucho más elevado de lo que en un principio pudiera haberse supuesto, debido al rango social de quienes los debían pagar, casi todos miembros de la oligarquía local. Es, por tanto, no sólo el alto índice de pago sino también la regularidad con que se pagan, en contraste con los otros tipos de censos.

El segundo lugar lo ocupan los censos cobrados a las villas de Elda, Elche y Sax por el disfrute del agua de la Fuente del Chopo con el 37,69 por ciento. Al comenzar nuestro período Villena debería haber percibido dos pensiones por censos a sus propios por el disfrute de este agua: una de 1.050 rs. que debia pagar Elda y otra de 500 rs. que debía satisfacer Elche. Sin embargo, desde la composición de las cuentas en 1708 ninguno de estos censos es pagado. Hasta 1727, fecha en que se produce una concordia entre Elda y Villena para el pago de los atrasos, sólo percibe dinero por este agua en dos momentos (1713 y 1726), pero en ambas ocasiones no son estas villas las que pagan sino la vecina villa de Sax por disfrutar durante cuarenta días del sobrante de este agua.

La citada concordia entre Elda y Villena, firmada el 2 de octubre de $1727^{(8)}$, con asistencia también de comisarios de Sax, consistía en la reducción de la deuda de 20.340 rs. a 8.775 rs. que debía pagar con una nueva pensión anual de 500 rs., que se sumaban a la anterior, además de un primer plazo de 1.275 rs. antes de cumplirse el año 1727. En el mismo contrato se expecificaba que Sax tenía derecho a la mitad del agua sobrante durante cuarenta días, pagando 300 rs. por ella.

Desde esa fecha sólo recibe Villena los 300 rs. a que se obligó Sax, junto con Elda, a pagar por ese agua sobrante, sin que lo hiciese Elda. Ante la morosidad de Elda, Villena se decide a cortar el suministro de este agua y ofrecérselo a Sax. Pero, ya que Elda no cumple su contrato, es Sax la que se va a beneficiar desde 1742 de este agua, en virtud de un contrato por el que se obliga a pagar 950 rs. por el agua de la Fuente del Chopo, más los cuarenta días sobrantes. Pero nuevamente se incumple este contrato, pues en 1749 Villena deja de percibir ingresos por este agua.

En cuanto a la pensión de Elche, desde la composición de las cuentas en 1708, no paga ninguna pensión, aunque Villena nunca apremia a su pago, probablemente conociendo que el agua era difícil que llegase a Elche tras su paso por toda la cuenca del Vinalopó, donde era tan necesaria.

El último lugar entre los censos lo ocupa el censo cobrado a los propios de Caudete $(21,99 \%)$, pero su importancia durante la corta época que se paga es de destacar puesto que el porcentaje es muy alto para sólo cobrarse durante quince años. 
Este proceso de impago y de nuevos contratos hace que se produzcan profundas variaciones en el movimiento de los ingresos percibidos por el municipio de Villena.

Estas profundas variaciones se cuantifican en el coeficiente de variación que asciende al 86,75 por ciento, el más alto de todos los grupos de ingresos de propios y el doble del que experimentan el conjunto de los ingresos de propios $(40,15 \%)$. Sobre todo son las variaciones en el pago de los censos por el agua las que provocan los saltos en el nivel de ingresos por este concepto.

\section{CUADRO IV}

\section{EVOLUCIÓN DE LOS INGRESOS POR LOS CENSOS}

\begin{tabular}{|c|r|r|r|}
\hline PERIODO & REALES & INDICE & TASA DE CRECIMIENTO \\
\hline $1708-12$ & 620 & 22,22 & - \\
$1713-17$ & 1.433 & 51,36 & 8,73 \\
$1718-22$ & 1.354 & 48,53 & $-0,56$ \\
$1723-27$ & 5.080 & 182,09 & 14,13 \\
$1728-32$ & 5.523 & 197,97 & 0,83 \\
$1733-37$ & 5.722 & 205,11 & 0,35 \\
$1738-42$ & 3.351 & 120,12 & $-5,20$ \\
$1743-47$ & 5.183 & 185,79 & 4,45 \\
$1749-53$ & 626 & 22,43 & $-19,05$ \\
$1754-59$ & 845 & 30,28 & 3,04 \\
$1760-66$ & 950 & 34,05 & 1,17 \\
\hline TOTAL .......... & 30.687 & & 1,63 \\
\hline
\end{tabular}

Fuente: A. M. V. «Libros de Propios» (elaboración propia).

Observamos cómo los momentos culminantes de estos ingresos se sitúan entre los años 1723-1747. Este período coincide, por una parte, con los años en que se paga el censo de Caudete y, por otra, con los que los censos por el agua son pagados con mayor regularidad, aunque en base a diferentes contratos.

Por lo tanto, las variaciones en el volumen de ingresos que el municipio percibe por este concepto no puede relacionarse con influjos externos, sino por el propio funcionamiento de sus componentes.

e) Las rentas por los sobrantes de rentas reales.

Estos sobrantes no siempre existían, teniendo incluso, a veces, que poner dinero el municipio, ya que el arrendador de alguno de estos efectos no había podido recaudar lo suficiente para cubrir el encabezamiento. Ello sucedió en Villena con el importe de las Penas de Cámara en 1763 y 1765 y con la hierba salada en 1763. 
Durante el corto período de nuestra serie los sobrantes ascienden a $\mathbf{1 2 . 1 7 0}$ rs. Entre éstos es el sobrante del aguardiente el que representa el mayor volumen con el 47 por ciento del total, seguido del sobrante de las Penas de Cámara con el 26,9 por ciento; en último lugar se sitúan los sobrantes de la hierba salada, que no tenía sus mejores momentos en esta época, pues se vio muy afectada por el descenso que experimentó el comercio durante la Guerra de los Siete Años.

f) Ingresos por alcances contra el mayordomo.

El particular funcionamiento de las cuentas de propios, en las que el mayordomo jugaba un papel destacado, hacía que en algunas ocasiones el dinero que recaudaba fuera superior a los gastos efectuados. En esos casos el dinero sobrante tenía que ser devuelto al año siguiente y se incorporaba a los caudales de ese año. Pero las dificultades que el mayordomo encontraba para la composición de las cuentas hace que no sólo nos debamos fijar en el resumen de las cuentas del año anterior, sino comprobar si efectivamente se incorporan al año siguiente.

El cuadro siguiente recoge lo que hemos podido comprobar con exactitud que sí entró en los propios como pago del mayordomo por sobrantes de las cuentas antecedentes.

\section{CUADRO V}

INGRESOS DE LOS PROPIOS POR ALCANCES A SU FAVOR

\begin{tabular}{|c|c|c|}
\hline PERIODO & REALES & INDICE \\
\hline $1708-12$ & - & - \\
$1713-17$ & 1.194 & 22,41 \\
$1718-22$ & 350 & 6,57 \\
$1723-27$ & 3.134 & 58,83 \\
$1728-32$ & 8.945 & 167,91 \\
$1733-37$ & - & - \\
$1738-42$ & - & - \\
$1743-47$ & 25.781 & 483,96 \\
$1749-53$ & 8.317 & 156,12 \\
$1754-59$ & - & - \\
$1760-66$ & 10.876 & - \\
\hline TOTAL.................. & 58.597 & -16 \\
\hline
\end{tabular}

Fuente: A. M. V. «Libros de Propios» (elaboración propia).

Observamos cómo estos ingresos se concentran en unas épocas muy determinadas, mientras que en otras apenas sí tienen importancia. Los mayores alcances a favor de los propios se sitúan entre 1743-53 y en el quinquenio final de 1760-66. 


\subsection{Evolución general de los ingresos de propios}

La evolución de estos ingresos estará determinada por la importancia de cada uno de los componentes de los propios y no se puede realizar un análisis de éstos sin tener en cuenta la evolución a lo largo de la serie estudiada del valor que cada uno de los grupos de propios adopta sobre el conjunto de los ingresos de propios.

En el conjunto de los ingresos de propios entre 1708-66 son las rentas por las propiedades inmobiliarias no agrícolas las que ocupan el primer lugar, suponiendo justamente el 50 por ciento $(50,03 \%)$. Las rentas por las propiedades inmobiliarias agrícolas no llegan a ocupar un tercio (el $30,91 \% 7$, por otra parte ya vimos las diferencias en la evolución de estos dos componentes de los propios, pues mientras el primero evoluciona claramente al alza, las rentas agrícolas casi se mantienen estables. Sin embargo, las rentas agrarias duplican al siguiente componente, los servicios públicos. Éstos suponen el 15,03 por ciento, mientras que el resto son componentes marginales, los censos con el 2,87 por ciento y los sobrantes de las rentas reales con el 1,14 por ciento.

La curva que refleja la evolución de los ingresos de propios, excluidos los alcances a favor del mayordomo para conocer fielmente las relaciones que existen entre este movimiento y la coyuntura económica general ${ }^{(9)}$, presenta un claro movimiento al alza con una tasa de crecimiento anual del 3,42 por ciento, pero con algunos movimientos bruscos tanto al alza como a la baja, lo que se refleja en que estos ingresos presentan un coeficiente de variación del 40,15 por ciento. Estos movimientos bruscos tienen, sin embargo, una característica común que es su escasa relevancia sobre la tendencia general, ya que tras una brusca subida se produce un descenso similar que hace volver el movimiento a la tendencia general.

Podemos distinguir tres períodos en dicho crecimiento. El primero abarcaría desde 1708 a 1727 en el que se produciría un movimiento general al alza mucho más elevado que en el resto de la serie. Este período tendría por lo tanto una tasa de crecimiento superior a la media de $1708-66(3,42 \%)$ con un crecimiento del 5,89 por ciento anual. A partir de 1728 entramos en la segunda etapa que perdura hasta 1746. Este período se caracteriza por su relativo estancamiento, aunque alterado por algunos ciclos bianuales de subidas y bajadas que no alteran el movimiento general al ser éstas de la misma cuantía que aquéllas. Su tasa de crecimiento es escasa, sólo del 0,84 por ciento, lo que atestigua este estancamiento.

El tercer y último período en el desarrollo de los ingresos de propios durante la época que tratamos se produce entre 1746 y 1766 . La tasa de crecimiento $(3,69 \%$ anual) se asemeja a la del conjunto del período $1708-66(3,42 \%)$, frente al crecimiento superior a la media del primer período y el inferior del segundo.

En el cuadro que recoge el movimiento quinquenal de estos ingresos se corrobora lo que hasta aquí hemos indicado y con él se atenúan las variaciones anuales, atendiendo más a la evolución a largo plazo. La alta tasa de crecimiento que se produce en el primer período se debe fundamentalmente a la incorporación pau- 
latina que en los bienes de propios se va experimentado, con unos bienes, además, que adquieren una gran importancia sobre el conjunto de los mismos como la tienda de saladura y especiería, la feria franca, el molino, etc. Por ello cuando dejan de producirse estas incorporaciones se produce un estancamiento. El siguiente movimiento claramente al alza, que se inicia en 1746, está motivado, por una parte, por la incorporación de un importante efecto de propios como son las balsas del cáñamo, pero también por el crecimiento que en casi todos los propios se produce en esta época.

\section{CUADRO VI}

EVOLUCIÓN DE LOS INGRESOS DE PROPIOS

\begin{tabular}{|c|c|c|c|}
\hline PERIODO & REALES & INDICE & TASA DE CRECIMIENTO \\
\hline $1708-12$ & 35.237 & 36,36 & \\
$1713-17$ & 48.826 & 50,39 & 3,31 \\
$1718-22$ & 64.861 & 66,94 & 2,88 \\
$1723-27$ & 86.996 & 89,79 & 2,97 \\
$1728-32$ & 89.757 & 92,64 & 0,31 \\
$1733-37$ & 100.356 & 103,58 & 1,12 \\
$1738-42$ & 104.943 & 108,31 & 0,44 \\
$1743-47$ & 107.297 & 110,74 & 0,22 \\
$1749-53$ & 123.897 & 127,87 & 1,44 \\
$1754-59$ & 141.950 & 146,51 & 1,36 \\
$1760-66$ & 161.663 & 166,82 & 1,30 \\
\hline TOTAL $\ldots . . . . . .$. & 1.065 .633 & & 3,42 \\
\hline
\end{tabular}

Fuente: A. M. V. «Libros de Propios» (elaboración propia).

El más importante de los gastos municipales sufragados por los propios era el compuesto por los salarios, pues en el conjunto de período que aquí tratamos (1708-66) representan el 35,59\% del total. Este capítulo de los gastos presenta una tasa de crecimiento muy alta $(4,80 \%)$, muy superior al del conjunto de gastos $(1,76 \%$ anual). Este crecimiento tiene un momento determinado en su desarrollo, se trata del periodo $1708-22$, debido a que se van agregando distintos funcionarios municipales.

El capítulo de las pensiones debía haber sido uno de los que mostraran un desarrollo más homogéneo a lo largo de la serie, pero el alto nivel de incumplimiento que manifiesta el municipio de Villena en ellos hace que varien en el tiempo. En él se incluyen tres censos (uno pagado a la familia Mercader, otro al Fisco de la Inquisición de Murcia y otro a las monjas agustinas de Almansa) -el 83\% del total de las pensiones-, los alquileres de alguna casa con el $4,81 \%$ y algunos 
«regalos», que se institucionalizan entre $1710-40$, ofrecidos a algunos funcionarios del concejo.

Los gastos religiosos representan un capítulo importante dentro de los gastos de propios, pues en el conjunto del período que aquí tratamos suponen el $16,90 \%$, aunque en algunos períodos supera el $20 \%$. Además estos gastos presentan un crecimiento muy superior al del resto de gastos de propios, pues mientras éstos crecen a razón de un 1,76\% anual, los gastos religiosos lo hacen un 2,11\%. Dentro de este capítulo de gastos son las fiestas religiosas de la ciudad las que suponen el mayor porcentaje. En el conjunto de la serie ocupan el 82,07\%. Las fiestas de carácter civil (funerales reales, celebración de victorias, etc.) son esporádicas y sólo representan el $5 \%$ de estos gastos. Las hemos incluido en este apartado porque en su mayoría están realizadas a base de actos religiosos como misas, sermones, etc. También forman parte de este apartado las limosnas $(3,2 \%)$ que el municipio otorgaba a los cabildos, conventos y pobres de la ciudad, pero también a los penitentes que circulaban por ella o la limosna que, por orden de Felipe V, tuvieron que pagar los municipios para la conservación del Santo Sepulcro de Jerusalem desde 1717. Por último aparecen las misas y rogativas que se celebraban en Villena, sobre todo, para hacerse acreedores de la gracia divina y evitar alguna epidemia o por la falta de agua. Este grupo supone el segundo apartado tras las fiestas religiosas con el $9,5 \%$.

Aunque el apartado dedicado a las obras públicas suponen dentro de los gastos de los propios una cifra importante $(9,00 \%)$ hay que hacer notar que gran parte de los que le surgen al municipio durante nuestro período no se sufragan por ellos sino que se consiguen realizar mediante una concesión real especial de su propio caudal. Nos referimos a los gastos causados por el paso de la Guerra de Sucesión por Villena entre 1705-1707 y que produjeron destrozos en el Ayuntamiento, la Torre del Reloj, la carnicería pública, las Cárceles, el Peso Real y el Pósito. Una vez realizadas estas obras el municipio apenas si va a dedicar parte de sus caudales para componer sus edificios. No será hasta el período 1721-26 cuando estos gastos se regularizan al tener el concejo que arreglar y dotar de algunas dependencias a los edificios públicos, limpiar las fuentes y realizar el amojonamiento del término.

Dentro de los gastos administrativos aparecen por una parte los llamados en la documentación «negocios de corte» (pleitos seguidos en la Corte, pago de confirmación de privilegios y de escrituras) y los gastos producidos por el desarrollo de la administración periférica, ya que el concejo de Villena tenía que hacer frecuentes viajes a Murcia, San Clemente o las ciudades próximas.

Estos ingresos suponen el $8,48 \%$, dominando los Negocios de Corte con el $67,2 \%$ de los gastos administrativos.

Los gastos militares que tiene que sufragar el municipio de Villena no son muy cuantiosos pues sólo suponen el 2,07\% del total de los gastos sufragados por los propios. El primer momento en que estos gastos son relativamente importantes es durante los años finales de la Guerra de Sucesión (1708-13) en los que 
tiene que atender al pago del viaje de los soldados repartidos a la ciudad para formación de una partida del ejército en Murcia, a la soldada ofrecida a los componentes de la milicia formada en Villena para ayudar al sitio de Alcoy y Jijona y los gastos ocasionados durante el tránsito de los ejércitos por la ciudad. El segundo período en que estos gastos son importantes surge en 1751, el motivo es el establecimiento desde 1749 de un cupo de soldados a cada municipio.

La Corona se servía de los municipios y de sus ingresos para conseguir los propios caudales. El rey comenzó a cobrar en 1711 un valimiento sobre el valor de las dehesas, que suponía el cobro de un tercio de los ingresos que el municipio obtenía por su arrendamiento. La creacion en 1760 de la Contaduría General de Propios y Arbitrios va a suponer un nuevo gasto al municipio pues se estableció que se debía contribuir para su funcionamiento con el dos por ciento de los ingresos de propios. Sin embargo, este capítulo no es de los más cuantiosos pues sólo supone el $2,15 \%$ del total de lo gastado por los propios.

El capítulo de gastos en deudas lo hemos dividido en dos grandes apartados: las deudas al mayordomo por haberlas suplido durante su mandato al no tener suficiente con lo que ingresaba por los propios, y las deudas a otros vecinos o instituciones. La primera es la más importante con el $61,2 \%$ de las deudas, y entre las segundas encontramos las que el municipio tiene que satisfacer tras la firma de la paz de Viena (1717) en concepto de pago por los bienes que durante la guerra y después de ella fueron confiscados a los vecinos de Caudete y Biar con propiedades en Villena. En conjunto suponen el 14,34\% del total de lo gastado por los propios.

En el apartado de otros gastos sólo tenemos el $1,8 \%$ y en ellos aparecen algunas partidas inclasificables porque no aparecen detallados sino que en una misma partida de gastos aparecen diversos efectos como tránsitos, salarios extraordinarios, pequeñas obras y compras de enseres. Otros se refieren a gastos extraordinarios que se producen en años muy concretos: en 1756 encontramos el gasto en sofocar un incendio en la Sierra de Salinas y, sobre todo, los 15.579 rs. gastados en 1757-58 en la extinción de la langosta.

Una vez que hemos detallado la importancia de cada uno de los componentes de los gastos municipales pasamos a conocer cómo se desarrollan el conjunto de los gastos a lo largo de la serie. Como observamos en la gráfica 2 los gastos sufragados por los propios presentan diversas fases en su evolución. Una primera fase se extendería desde 1708 a 1723 , caracterizada por un fuerte crecimiento, a razón de un 7,09\% anual. La razón se encontraría en que tras la composición de las cuentas en 1708 no todos los oficiales del concejo aparecen, por lo que su paulatina incorporación al mismo hace que crezca el volumen de los salarios. Otra causa importante sería que durante esta primera época al ser los gastos superiores a los ingresos existía una deuda con el mayordomo que los suplía.

La segunda etapa comprendería desde 1724 a 1750 y se opondría a la primera parte por la relativa estabilidad que presentan los gastos, aunque con algunos movimientos cíclicos bianuales. Su crecimiento sería negativo, pero tan sólo de $-0,26 \%$ anual. 
El tercer período se situaría entre 1750 y 1766 con una fase ascendente (175056) y una fase descendente desde 1756 al final de nuestra serie. La fase de ascenso se produce por el crecimiento producido en unos años determinados $(1751,1753$, 1756) del gasto de los salarios, las deudas y la aparición del pago del encabezamiento de la sosa o barrilla y las Penas de Cámara y el auge de las obras públicas. El descenso se produce paulatinamente, pero los años críticos son 1764-65. Ello produce que el año 1765 vuelva a presentar el mismo volumen de gasto que en 1728 y la media de la primera fase. Este descenso es casi generalizado pues afecta a casi todas las partidas. Esto haría coincidir esta fase de profundo descenso en los gastos con la creación de la Contaduría General de Propios y Arbitrios, sin embargo, no podemos documentar que exista una relación directa entre ambos hechos, aunque debemos considerar que una de las razones de tal organismo fue contener el gasto desproporcionado de algunos municipios.

El cuadro que agrupa los datos quinquenales de los gastos refleja también lo que hemos indicado con la única salvedad de que el segundo período se extendería hasta el quinquenio 1723-27. Según este cuadro el crecimiento del primer período se produciría fundamentalmente en el quinquenio 1723-27, mientras que el tercer período presentaría la fase ascendente en el gasto entre 1749-59 y la fase descendente entre $1760-66$ con una tasa de decrecimiento anual de $-4,4 \%$, la más alta de toda la serie.

CUADRO VII

EVOLUCIÓN DE LOS GASTOS DE PROPIOS

\begin{tabular}{|c|c|c|c|}
\hline PERÍODO & REALES & INDICE & TASA DE CRECIMIENTO \\
\hline $1708-12$ & 56.638 & 55,30 & - \\
$1713-17$ & 68.642 & 67,07 & 1,94 \\
$1718-22$ & 69.255 & 67,67 & 0,08 \\
$1723-27$ & 97.413 & 95,19 & 3,47 \\
$1728-32$ & 94.490 & 92,33 & $-0,30$ \\
$1733-37$ & 118.441 & 115,74 & 2,28 \\
$1738-42$ & 113.262 & 110,68 & $-0,44$ \\
$1743-47$ & 102.456 & 110,12 & $-0,99$ \\
$1749-53$ & 119.131 & 116,41 & 1,51 \\
$1754-59$ & 174.922 & 170,93 & 3,90 \\
$1760-66$ & 111.010 & 108,47 & $-4,40$ \\
\hline TOTAL........ & 1.125 .660 & & 1,23 \\
\hline
\end{tabular}

Fuente: A. M. V. «Libros de Propios» (elaboración propia). 
El peculiar sistema en que se basaba el funcionamiento de los propios hace que un análisis del balance general entre gastos e ingresos tenga unas características y unas claves de interpretación bien diferentes a las que pueden observarse en otros estudios hacendísticos.

El déficit que se producía en algunos años apenas si influía en el desarrollo posterior del caudal de los propios ya que no era acumulativo si no que al tenerlo que pagar el mayordomo de su caudal se incorporaba a las cuentas del año siguiente como hemos indicado. A ello contribuía el hecho de que el mayordomo se sucediese en el cargo lo que hacía fácil cobrar la deuda, ya que era el mismo quien tenía que pagársela al año siguiente.

Como refleja el gráfico 3 , podemos dividir el movimiento de estos caudales en dos grandes etapas: una abarca desde 1708 a 1740 y otra desde 1741 al final de la serie. Si la primera se caracteriza por el contínuo déficit, interrumpido en cortos períodos, la segunda lo estaría por tener dos períodos a su vez, de superávit en los propios entre los que se sitúa una nueva época de déficit.

Quizá el cambio que se produce desde 1741 podramos relacionarlo con la aparición como vimos de un importante efecto de propios como eran las balsas del cáñamo, produciéndose el nuevo cambio hacia la aparición del déficit en 1750 por las contínuas deudas con el mayordomo, el incremento de los salarios y la aparición del encabezamiento de la sosa y de las Penas de Cámara. En 1757 vuelve a aparecer el superávit, que se produce por el ya comentado descenso de los gastos y por el incremento de los ingresos, sobre todo de las propiedades inmobiliarias no agrícolas. 


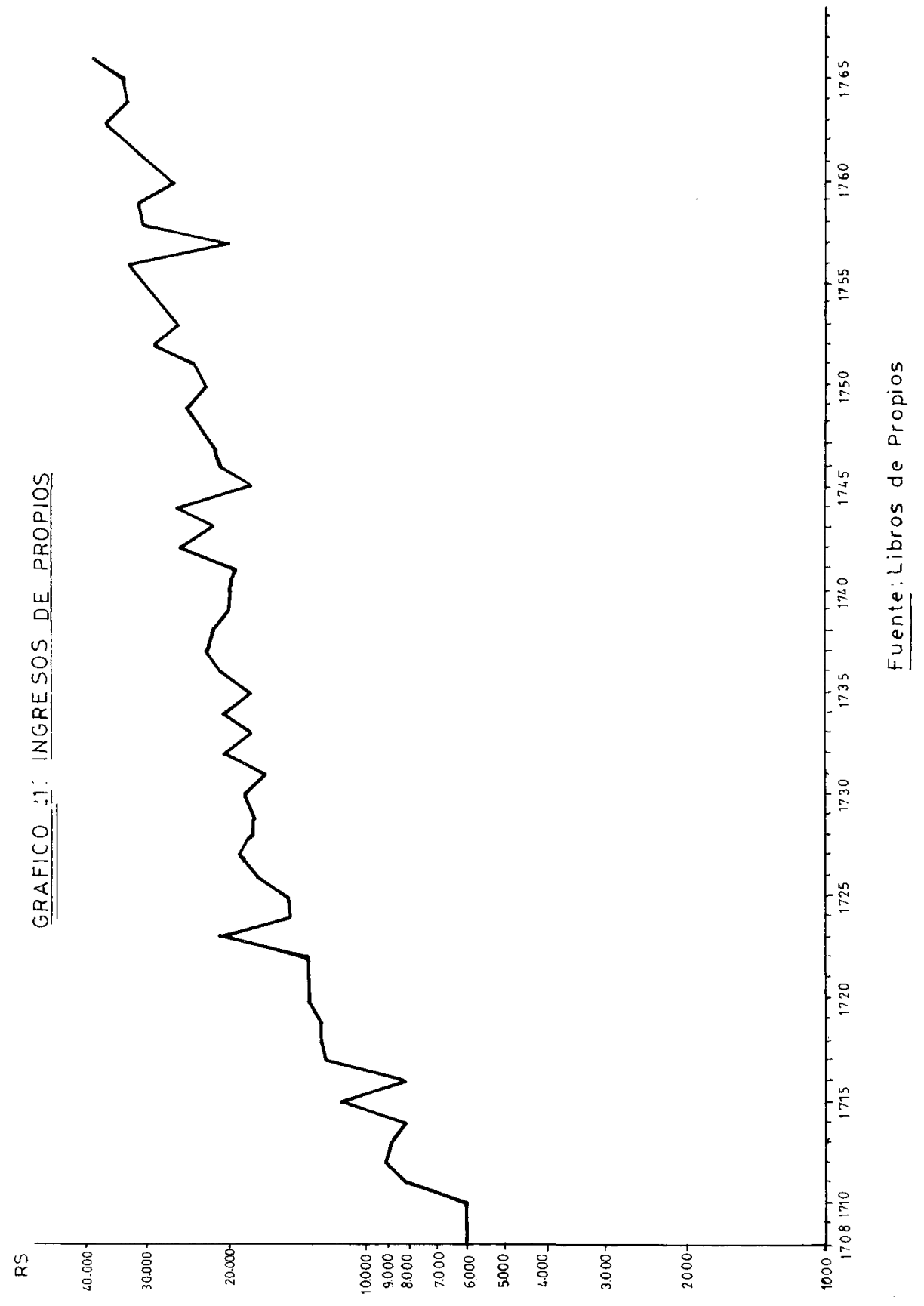




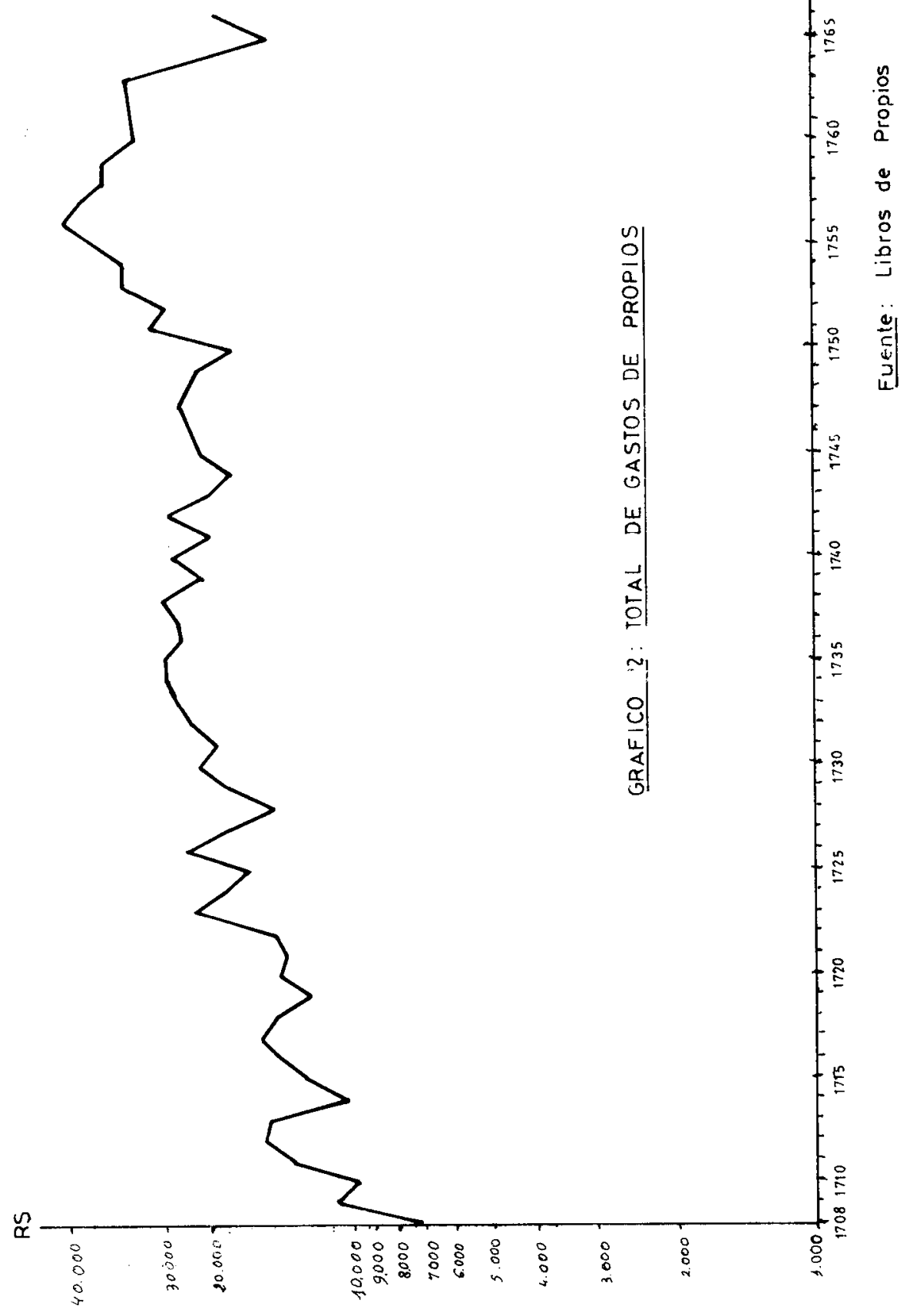




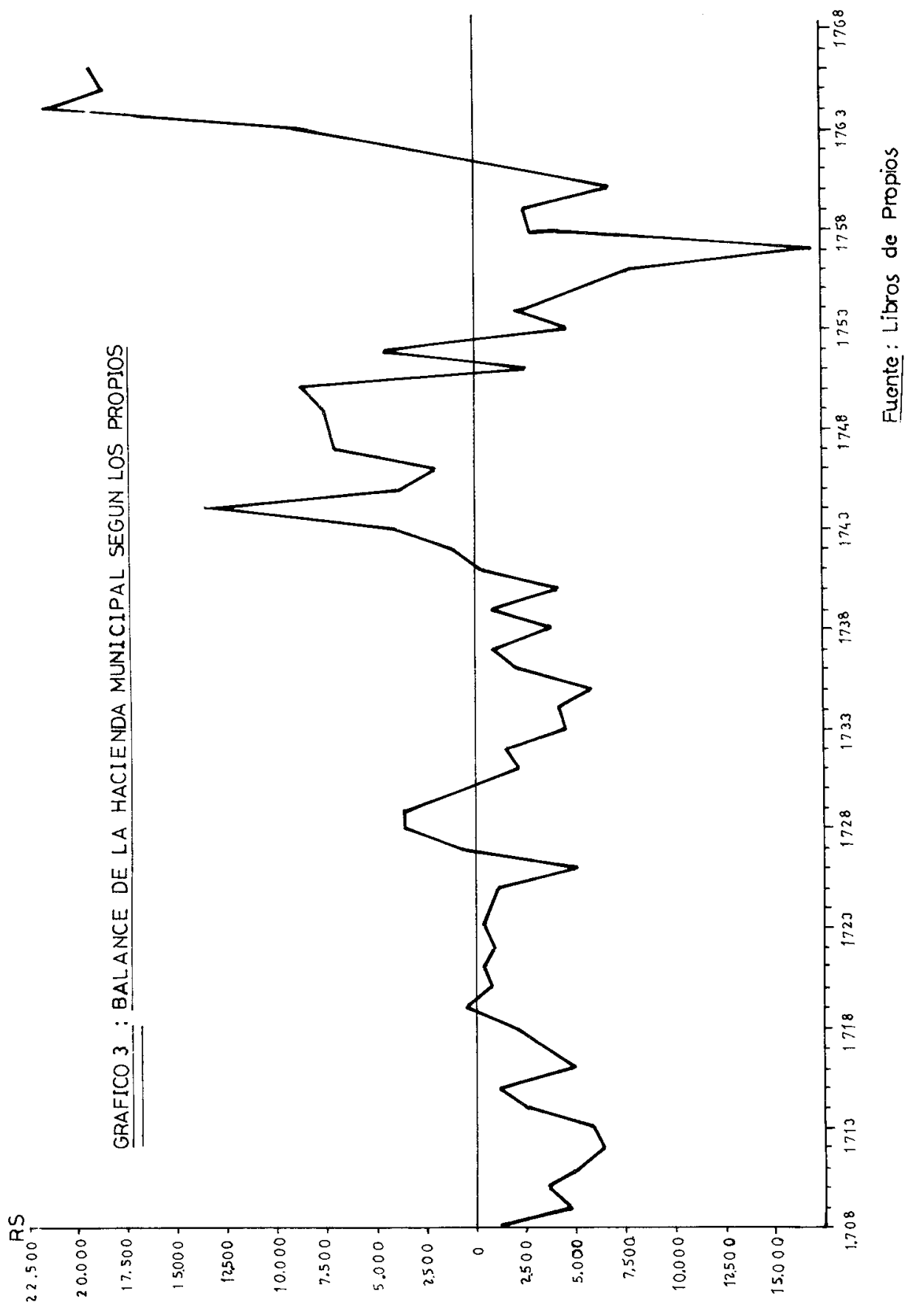




\section{NOTAS}

(1) La presente comunicación se basa en parte de nuestra Memoria de Licenciatura, que con el título «La hacienda municipal de Villena durante la primera mitad del siglo XVIII (1708-I766)», dirigida por el Dr. Enrique Giménez López fue leida el 8 de octubre de 1986 en la Facultad de Filosofía y Letras de la Universidad de Alicante.

(2) «Esta ciudad ahora ni desde hace mucho tiempo administra ni tiene ningún tipo de arbitrios», A. M. V, Acuerdos Municipales (A. M.), sesión 18 de marzo de 1735.

(3) HERNÁNDEZ MARCO, J. L.: Propiedad de la tierra y cambio social en un municipio fronterizo: Villena (1750-1888). Alicante, 1983, Ap. Doc. p. 159.

(4) Novísima Recopilación, 6, 16, 13, cap. 13.

(5) Los quinquenios han sido agrupados atendiendo a la existencia de datos, por ellos algunos tienen intervalos superiores a cinco años.

(6) Para la tasa de crecimiento se ha utilizado el método del interés compuesto y para el coeficiente de variación la relación entre la desviación estandar y la media.

(7) El índice 100 representa la media de todos los años.

(8) No se conserva en el A. M. V. el texto de la concordia, pese hace un resumen de ella en las cuentas de 1729 .

(9) Hemos adotado el sistema de representación semilogarítmico por considerarlo más apropiado a los estudios hacendísticos ya que comparan proporcionalmente los datos, apreciando los movimientos acumulativos. 